PALEO

Revue d'archéologie préhistorique

$27 \mid 2016$

Varia

\title{
La grotte de Rouffignac (Dordogne, France) : un gîte de matière première exploité au Mésolithique
}

Rouffignac cave (Dordogne, France) : a raw material site exploited during

Mesolithic

Morgane Dachary, Frédéric Plassard et Hélène Valladas

\section{OpenEdition}

Journals

Édition électronique

URL : http://journals.openedition.org/paleo/3202

DOI : 10.4000/paleo.3202

ISSN : $2101-0420$

Éditeur

SAMRA

Édition imprimée

Date de publication : 30 décembre 2016

Pagination : 133-148

ISSN : 1145-3370

Référence électronique

Morgane Dachary, Frédéric Plassard et Hélène Valladas, « La grotte de Rouffignac (Dordogne,

France) : un gîte de matière première exploité au Mésolithique », PALEO [En ligne], 27 | 2016, mis en

ligne le 01 juin 2018, consulté le 07 juillet 2020. URL : http://journals.openedition.org/paleo/3202 ; DOI : https://doi.org/10.4000/paleo.3202

\section{(c) $($ () $\odot$}

PALEO est mis à disposition selon les termes de la licence Creative Commons Attribution - Pas d'Utilisation Commerciale - Pas de Modification 4.0 International. 


\title{
La grotte de Rouffignac (Dordogne, France) : un gîte de matière première exploité au Mésolithique
}

\author{
Morgane DACHARY(a), Frédéric PLASSARD ${ }^{(b)}$, Hélène VALLADAS ${ }^{(c)}$
}

\begin{abstract}
Résumé : La fouille d'un secteur restreint d'une galerie profonde de la grotte de Rouffignac documente une exploitation des nodules de silex directement accessibles sur les parois, le plafond et dans le remplissage argileux de la cavité. L'étude technologique des vestiges lithiques ainsi que les remontages permettent de reconstituer les comportements des Hommes. La série, très homogène, témoigne du test, du dégrossissage et parfois du débitage sur place des nodules. Aucun des supports produits n'est retouché, aucun percuteur n'a été retrouvé et aucun vestige ne documente une autre activité que la taille. Cependant, les caractéristiques techniques des vestiges et une datation radiocarbone obtenue sur charbon de bois conduisent à associer le locus étudié aux niveaux mésolithiques mis au jour sous le porche d'entrée (Barrière 1973a, b, 1974). En outre, un examen rapide du sol de certaines galeries révèle que le locus étudié est l'illustration d'un comportement récurrent dont la grotte a conservé le témoignage.
\end{abstract}

Mots-clés : Mésolithique, technologie lithique, exploitation de la matière première, Grotte de Rouffignac.

Abstract: Rouffignac cave (Dordogne, France): a raw material site exploited during Mesolithic. The excavation of a small area of a deep gallery within Rouffignac cave produced evidence for the exploitation of flint nodules accessible in the walls, ceiling and clayey fill of the cavity. A technological analysis of the lithic artifacts combined with refitting shed new light on the behavior of the groups who occupied the cave. The very homogeneous lithic assemblage demonstrates the on-site testing, roughing-out and sometimes reduction of nodules. No retouched blanks or hammerstones were recovered, and there is no evidence for any activity other than knapping. However, the technological characteristics of the assemblage and a radiocarbon date obtained on charcoal are consistent with the Mesolithic levels documented at the cave's porch (Barrière 1973a, b, 1974). Finally, a rapid survey of the floor of certain galleries shows the behavior documented in the studied area to be repeated feature of the cave's occupation.

Key-words: Mesolithic, lithic technology, raw material exploitation, Rouffignac cave.

L'exploitation des matières premières siliceuses par les Hommes de la Préhistoire est largement documentée pour des gîtes de surface à toutes les périodes préhistoriques et par le creusement de mines, surtout au Néolithique. L'exploitation de silex directement accessibles dans des galeries naturelles, creusées dans des calcaires à silex, n'est que rarement signalée (Gopher et Barkai 2006 ; Apellaniz et Domingo Mena 1987). Le Mésolithique de la grotte de Rouffignac présente donc une originalité jusqu'ici peu renseignée.

(a) Université Toulouse-Jean Jaurès, UMR 5608, TRACES, Maison de la recherche, 5 allée Antonio Machado, FR-31058 Toulouse cedex - morgane.dachary@orange.fr

(b) Université de Bordeaux, UMR 5199, PACEA, Allée geoffroy Saint-Hilaire, CS 50023, FR-33615 Pessac cedexfrederic.plassard@wanadoo.fr

(c) Laboratoire des Sciences du climat et de l'environnement LSCE/IPSL, CEA-CNRS-UVSQ, Avenue de la Terrasse,

FR-91198 Gif-sur-Yvette cedex - helene.valladas@Isce.ipsl.fr 
Située sur un affluent secondaire de la rive droite de la Vézère, en Dordogne, et d'abord connue comme une grotte ornée, riche de 250 représentations pariétales paléolithiques (Barrière 1982 ; Plassard J. 1999 ; Plassard F. 2005a), la grotte de Rouffignac fut aussi largement fréquentée au cours de l'Holocène. Les travaux de $\mathrm{Cl}$. Barrière documentent des occupations mésolithiques sous le porche d'entrée ainsi que la fréquentation protohistorique de la grotte à des fins sépulcrales (Barrière 1973a et b, 1974 ; Chevillot 1981). L'identification de silex taillés dans la partie profonde de la grotte remonte aux premières prospections archéologiques au cours de l'été 1956 (Nougier et Robert 1957 - p. 99). Par la suite, $\mathrm{Cl}$. Barrière signala des « ateliers de taille » en plusieurs points de la caverne et les attribua au Mésolithique, les mettant en relation avec les occupations mises au jour sous le porche d'entrée. Pour autant, il ne réalisa aucune étude détaillée de ces vestiges. Plus récemment, dans le cadre d'une prospection systématique concernant le sol des galeries ornées et visant à mettre au jour des vestiges mobiliers liés $\mathrm{au}(\mathrm{x})$ passage(s) des artistes paléolithiques (Plassard 2005a et b), nous nous sommes intéressés à un petit amas de taille situé au pied d'une paroi ornée.

Le matériel prélevé réunit plus de 1200 vestiges parmi lesquels $95 \%$ de silex qui font l'objet ici d'une étude technotypologique. Des datations radiocarbone sur charbons de bois permettent en outre de restituer le matériel dans le contexte chronologique des occupations du porche d'entrée. Cette série pose aussi la question des activités pratiquées sur place, dans un milieu relativement hostile où la gestion de l'éclairage, et plus généralement la logistique, tiennent une place particulière. Outre la taille, les vestiges présents documentent-ils d'autres activités, comme c'est souvent le cas dans les sites d'atelier mis au jour et fouillés à l'extérieur? D'un autre point de vue, cette étude amène à la réévaluation du potentiel archéologique du sol des galeries de Rouffignac, trop longtemps sous-estimé pour cause de fréquentations historiques du réseau.

\section{1 - Présentation du locus}

Creusé pour l'essentiel dans les calcaires à silex du Santonien supérieur (Platel 1999), le réseau karstique de Rouffignac offre environ huit kilomètres de galeries réparties en trois étages. Excepté deux diverticules des étages inférieurs, seul le niveau supérieur présente un intérêt archéologique. Totalement fossile, il est constitué de galeries assez vastes où affleurent d'innombrables nodules de silex. Ces derniers sont également abondants dans les remplissages argileux qui colmatent partiellement les couloirs.

Situé à environ $800 \mathrm{~m}$ de l'entrée, le secteur fouillé se trouve dans la partie profonde de la galerie Henri Breuil, dans un secteur non concerné par les aménagements touristiques (fig.1a). Après une zone dont le sol est très tourmenté par les bauges d'ours, puis un soutirage vers les niveaux inférieurs, un secteur au sol plus régulier se développe sur une dizaine de mètres de longueur. À cet
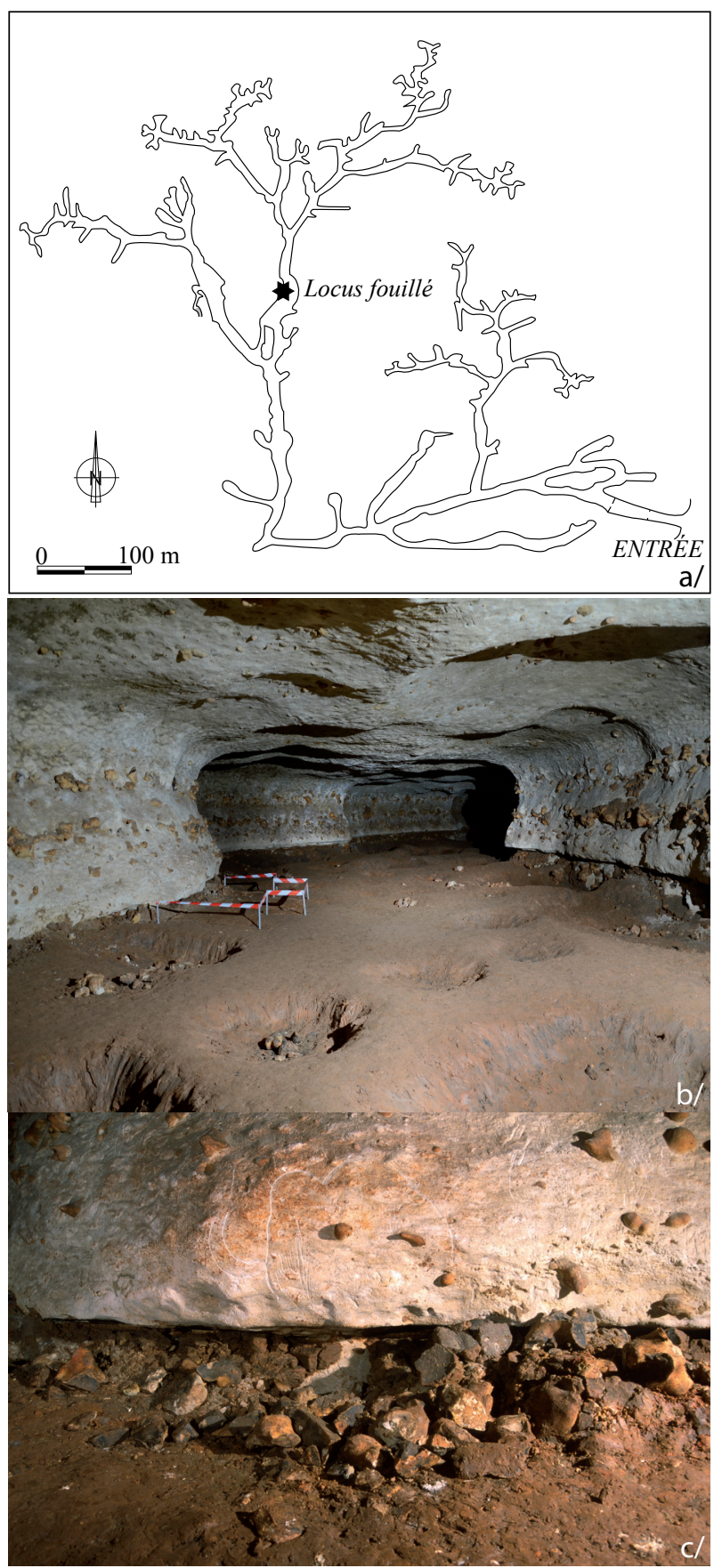

Figure 1 - a- Plan de la grotte et localisation du locus étudié. b-Vue générale de la galerie ; la zone étudiée est balisée sur le côté gauche. c- L'amas de taille visible au pied de la paroi ornée, avant son démontage.

Figure 1 - a- Plan of Rouffignac cave and location of the study area. $b$ - General view of the gallery with study area indicated on the left. $c$ - The knapping cluster at the base of the decorated wall before the excavation. 
endroit, la galerie a 5 à $6 \mathrm{~m}$ de large et 2,20 $\mathrm{m}$ à 2,50 $\mathrm{m}$ de haut (fig. 1b).

À la base de la paroi gauche, un artiste paléolithique a gravé une silhouette de mammouth ( $n^{\circ} 225$ de l'inventaire de $\mathrm{Cl}$. Barrière 1982). C'est immédiatement au pied de cette représentation que se trouvait un petit amas d'éclats et de blocs de silex auxquels étaient associés des charbons de bois. Plusieurs dizaines de vestiges étaient directement visibles sur le sol sur une surface à peine supérieure à un mètre carré (fig. 1c).

\section{2 - Contexte sédimentaire et taphonomique, méthode de prélèvement}

Le contexte sédimentaire et taphonomique de ce locus, et plus généralement des vestiges susceptibles d'être identifiés à Rouffignac (excepté dans la zone du porche), est marqué par une quasi absence de sédimentation. En effet, nous sommes confrontés à seulement deux types de sol tout aussi fossiles l'un que l'autre: des zones de grands éboulis probablement plus anciens que les premières fréquentations humaines, et des sols argileux creusés de bauges par des ours qui avaient abandonné la grotte longtemps avant le passage des artistes paléolithiques. Par conséquent, les vestiges archéologiques se trouvent uniquement en surface et en dehors de toute stratigraphie. En outre, l'histoire complexe de la caverne, sans cesse visitée depuis des millénaires, oblige à s'interroger sur le degré de perturbation des ensembles de vestiges, sachant que le facteur anthropique est dans ce cas le plus important.

L'ensemble de ces observations nous a conduits à adapter les méthodes de prélèvement à ce contexte original. Si de telles conditions facilitent la prospection, elles obligent à nuancer la notion de fouille. Les interventions in situ se traduisent essentiellement par un prélèvement direct du matériel parfois après un très léger décapage. Par ailleurs, il est apparu au cours de l'opération de terrain que si les plus grosses pièces étaient assez mobiles, et qu'il était donc difficile d'affirmer qu'elles se trouvaient en position primaire, en revanche, les plus petites, enfoncées de quelques millimètres dans l'argile très compacte du sol, avaient peu de chance de s'être beaucoup déplacées. C'est pour cette raison que nous avons essayé de coter tous les vestiges, sans limite de dimension. Le faible volume de sédiment prélevé et isolé par quart de mètre carré, a néanmoins été tamisé sous l'eau et l'ensemble des refus de tamis a été conservé et trié. Des esquilles prises dans l'argile collée aux pièces cotées sont apparues au cours du lavage du matériel et ont, elles aussi, été conservées.

Les décapages n'ont entamé le sédiment que sur un à deux centimètres. Cependant un test de fouille plus profonde (six centimètres) conduit sur un quart de mètre carré a montré le caractère totalement stérile de l'argile sous-jacente.

\section{3 - Les vestiges archéologiques}

\section{1 - Présentation générale}

Le matériel recueilli se répartit inégalement entre vestiges lithiques, charbons et esquilles osseuses. Quantitativement, l'industrie lithique domine largement (1 184 pièces cotés soit $95 \%$ des vestiges récoltés) alors que seuls 50 charbons ont été prélevés et qu'il n'y a que six esquilles osseuses, toutes indéterminées, dont l'altération laisse penser qu'elles sont en relation avec le passage des Ours des cavernes et non avec celui des Hommes.

La surface décapée est assez régulière, quoique affectée d'un léger pendage vers l'axe de la galerie. Elle présente en outre trois accidents d'ampleurs limitées (fig. 2). Une fente de dessiccation traverse obliquement les carrés A2, B2 et B1. Elle est surtout visible en $A 2$, où elle a peut-être été accentuée par l'extraction d'un nodule pris dans l'argile. Les deux autres sont les dépressions peu profondes (une dizaine de centimètres au maximum) qui affectent les carrés B2 et C2. Ce dernier est d'ailleurs en dehors de la zone fouillée, mais les grosses pièces réunies dans la petite cuvette ont été prélevées sans cotation et intégrées à l'étude du matériel. Le fond de la dépression de B2 s'est avéré pauvre en silex, mais induré et riche en charbons. Un prélèvement micromorphologique a permis la réalisation d'une lame mince qui montre des traces de rubéfactions (C. Ferrier, in verbis). Cette dépression probablement naturelle a sans doute accueilli un petit foyer d'éclairage.

Ce tableau laisse facilement comprendre que l'étude des vestiges lithiques recueillis constitue la source d'information essentielle pour répondre aux questions posées.

\section{2 - Étude technologique du matériel lithique}

\subsection{1 - Méthodologie}

La méthodologie mise en œuvre s'est organisée autour de deux pôles : une analyse technologique approfondie et la réalisation de remontages physiques, au sein de l'ensemble de la série prélevée en fouille mais aussi avec les nodules fracturés sur la paroi.

L'étude technologique s'est accompagnée d'une saisie systématique de certains éléments descriptifs, facilitant la diagnose de la technique de percussion : présence/absence du talon, nature du talon, présence/absence d'esquille bulbaire, d'accident de taille ou d'ondulations marquées sur la face inférieure.

\subsection{2 - Caractéristiques générales de la série}

Les pièces cotées lors de la fouille sont essentiellement des éclats $(43,9 \%)$ puis des esquilles $(25,8 \%)$ et des fragments techniquement non caractéristiques (19,7\%). Ces deux dernières catégories (soit $45,5 \%$ des vestiges) sont dépourvues des stigmates techniques qui permettraient de les restituer dans un schéma opératoire. Cette absence s'explique soit par la dimension très réduite de la pièce soit par l'abondance des fracturations naturelles révélées par la fragmentation du bloc. 


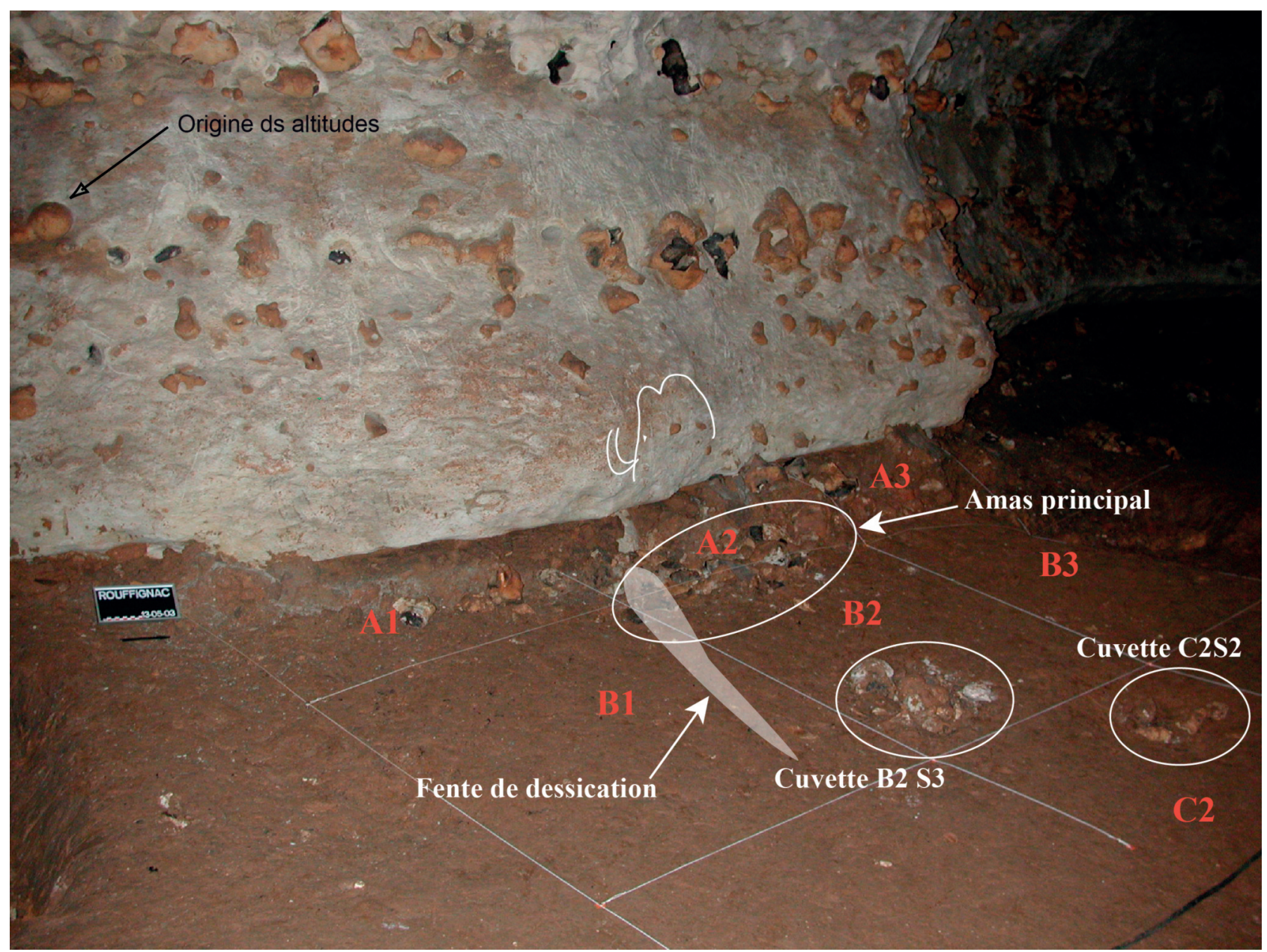

Figure 2 - Vue générale du locus avant la fouille.

Figure 2 - General view of the study area before excavation.

Les $10 \%$ restant du matériel sont répartis, par ordre décroissant d'importance, entre les esquilles bulbaires (entières ou fragmentaires : 4,4\%), les nodules plus ou moins fragmentaires $(3,6 \%)$, les éclats de préparation du talon $(0,7 \%)$ et les éclats allongés $(0,7 \%)$. Ébauche de nucléus à lamelles, éclats de préparation de nucléus sur tranche d'éclat et lamelles sont rarissimes.

Toutes catégories confondues, le taux de cortex est généralement inférieur à $15 \%$, même si les pièces couvertes de $80 \%$ ou plus de cortex représentent une part non négligeable de la série.

Dans le détail, pour les pièces de dimension maximale inférieure ou égale à $1 \mathrm{~cm}$, le taux de cortex est dans $80 \%$ des cas inférieur à $15 \%$, alors que ce taux est de $50 \%$ pour les pièces de plus de $1 \mathrm{~cm}$. De même, un taux de cortex supérieur ou égal à $80 \%$ concerne $28 \%$ des pièces de plus de $1 \mathrm{~cm}$ contre à peine $10 \%$ des pièces de dimension inférieure ou égale à $1 \mathrm{~cm}$.
Enfin, parmi les éclats, quelque soit leur dimension, 63,2\% sont couverts de moins de $25 \%$ de cortex, $14 \%$ sont semicorticaux et $22,8 \%$ sont corticaux.

\subsection{3 - Analyse des stigmates de percussion}

Outrepassages et cassures Siret concernent uniquement les éclats : 13 pièces dans le premier cas, 49 dans le second. $15,1 \%$ des éclats sont rebroussés (81 pièces, ainsi qu'un éclat Kombewa et une lamelle). Enfin, seules deux pièces ont une préparation soigneuse du talon : il s'agit d'un éclat et de l'unique éclat de cintrage décrit.

Les éclats d'entame (corticaux ou semi-corticaux) sont souvent soit clairement rebroussés, soit avec deux ou trois série d'ondes marquées en extrémité distale.

II faut noter l'abondance des micro-esquilles en forme de bâtonnets de très petites dimensions (pas plus de $2,5 \mathrm{~cm}$ de longueur maximum). Leur présence est la conséquence de la forte fissuration par diaclase de la matière première et non le signe d'une ouverture des blocs par percussion 
bipolaire sur enclume. En effet, d'une part, les pièces présentant des négatifs de percussion violente montrent que ces négatifs sont toujours orientés dans la même direction et jamais en opposition et, d'autre part, certains blocs fortement diaclasés explosent littéralement à l'ouverture, provoquant le dégagement d'une multitude d'esquilles (fig. 3a).

L'abondance des micro-esquilles peut être aussi la conséquence de l'irrégularité de la matière première comme de la force nécessaire pour passer à travers une zone sous-corticale parfois très molle, pouvant atteindre $2 \mathrm{~cm}$ d'épaisseur.

Étant donné le contexte de fréquentation de la grotte, le mode de percussion doit être discuté avec attention, d'autant qu'il peut constituer un élément de datation s'il s'agit de métal ou de percussion au punch. La rareté des lèvres sous le talon, l'abondance des accidents Siret $(9,2 \%$ des éclats), des rebroussés (15,1\% des éclats), des talons

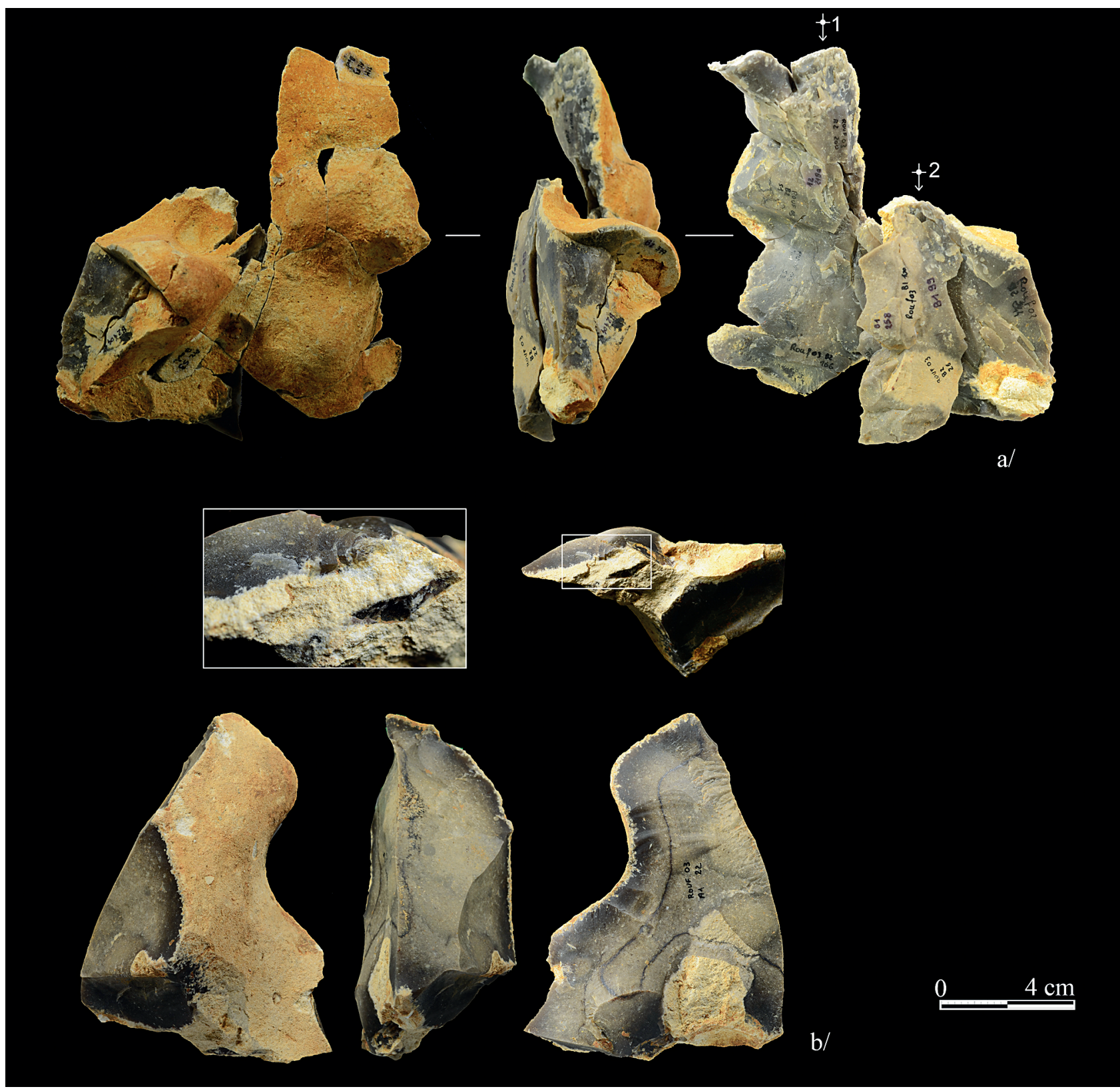

Figure 3 - a- B1\#53. Deux éclats de dégrossissage. Nettoyage de la surface d'un bloc fortement diaclasé. b- A1\#22, éclat cortical. Talon en haut à droite : surface diaclasique avec fissurations concentriques (Trois cônes incipients emboîtés).

Figure 3 - a- B1\#53. Two roughing-out flakes. Surface management of heavily jointed nodule- C2\#5. b- A1\#22, cortical flake. Butt, top right: natural (joint) surface with concentric cracks (Three overlapping incipient cones). 
lisses et larges (un seul est concave), des esquilles bulbaires et/ou des ondulations marquées sur la face inférieure nous font pencher pour un débitage perpendiculaire à la pierre dure.

Parallèlement, les cônes incipients peuvent être détourés sur pratiquement leur moitié. Ils ont des dimensions très variables : de 1-2 mm à 6-7 mm. Une pièce (A1\#22, fig. $3 b)$ montre que le percuteur s'est écrasé au moment de l'impact, provoquant la formation de trois cônes concentriques et imbriqués.

La fouille n'a pas permis de collecter de percuteurs : aucun nodule ne porte de trace d'utilisation comme percuteur (pas d'étoilement sur les surfaces) et aucun galet n'a été découvert.

\subsection{4 - Outillage}

Cette série ne comporte aucun outil. Les seules traces d'esquillements sur les tranchants correspondent à des ébréchures au moment du débitage.

\subsection{5 - Remontages}

Parmi les 902 pièces cotées de dimension maximale supérieure ou égale à $1 \mathrm{~cm}, 207$ ont pu être rattachées à un remontage (soit un taux de remontage de 22,9\%). Ajoutons que 12 pièces de dimension maximale inférieure à $1 \mathrm{~cm}$ ont été rattachées à ces mêmes remontages.

L'analyse des remontages physiques réalisés conduit à formaliser un schéma d'exploitation de la matière première (fig. 4), d'où il ressort que les prélèvements se font par fracturation des blocs en parois ou par ramassage (et extraction ?) au sol. Un diagnostic de la qualité de la matière première par test est ensuite réalisé.

Si la matière première est de très bonne qualité, le bloc est alors transporté hors du locus étudié après abandon de pièces isolées (A2\#238, fig. 5c) ou témoignant de courtes séquences de débitage. Un dégrossissage, éventuellement suivi d'un début d'exploitation, peut aussi être entrepris sur place. II aboutit à l'abandon de la coque externe du bloc (A2\#56, fig. 6 ; B2\#96, fig. 5d et C2\#5, fig. 5a), et parfois d'éclats de mise en forme des convexités du nucléus (A2\#56 et B1\#107, fig. 7a).

La mauvaise qualité de la matière première se manifeste par l'absence de silicification interne, par une fissuration naturelle importante, ou par la présence de zone non exploitable (corne, cortex épais, zones diaclasées, ...). Dans les deux premiers cas, les tests sont poussés plus ou moins loin (A1\#21; A2 \#26, fig. 8b ; A2\#239, fig. 8d ; $A 3 \# 21$; B2\#87, fig. 8c et A2\#233, fig. 8a) et aboutissent à l'abandon sur place de tous les produits. Dans le dernier cas, le nettoyage est entrepris et conduit à l'abandon des zones non exploitables (A2\#109; A2\#20, fig. 8e). Une exploitation peut ensuite être entreprise avec succès ou non (B1\#54; B1\#93).
Les tentatives de raccords sur les parois ont été tentées pour les remontages réalisés au laboratoire et pour les plus gros éclats collectés à la fouille. Chaque remontage a été testé sur les négatifs visibles sur les deux parois de la galerie sur une quinzaine de mètres de part et d'autre de la zone fouillée.

Six pièces, ou groupes de pièces, ont pu être raccordées à la paroi. Dans tous les cas, ils se situent dans la zone immédiatement au-dessus du secteur de fouille. De gauche à droite, il s'agit de :

1) A1\#21, une branche de nodule dont l'extrémité opposée n’a pas été percutée ;

2) A2\#109 et le remontage A2\#20. Après l'enlèvement de la corne A2\#109, le tailleur a poursuivi le test par l'élimination des vestiges remontés autour de A2\#20 ;

3) A2\#239, sur le même nodule, mais la branche à l'extrême droite. L'extrémité opposée à la paroi n'a pas été percutée ;

4) au-dessus, le remontage A2\#233 qui s'accroche à la paroi par A2\#234. II s'agit d'un nodule avec une corne, testé, mais de mauvaise qualité ;

5) Enfin, à l'extrême droite de la paroi : A3\#21, une branche de nodule dont l'extrémité opposée n'a pas été percutée.

En d'autres termes, les blocs prélevés en parois n'ont presque jamais conduit à un réel débitage.

Les remontages très partiels et une grande partie des pièces isolées témoignent d'une présence majoritaire de courtes séquences de gestes techniques. Celles-ci documentent, dans leur immense majorité, le test de la matière première et le dégrossissage des blocs. Quelques blocs témoignent cependant de tentatives de production sur place de lamelles et de lames.

Les nucléus $A 1 \# 38, A 2 \# 22$ et B1\#93 (fig. 7b, 7d et 7c) illustrent des tentatives de production de lamelles aux dépens de dièdres présentant naturellement les angulations favorables. Une première lamelle est ainsi détachée en surface de l'éclat semi-cortical A2\#22 (fig. 7d), après aménagement d'une crête à un pan puis l'objet est abandonné. Pour le remontage A1\#38 (fig. 7b), la lamelle B1\#54 suit l'arête entre une surface diaclasique et le négatif d'un éclat, avant l'enlèvement d'au moins quatre éclats d'aménagement du plan de frappe. Si les deux premiers sont des ébauches, le troisième (B1\#93, fig. $7 \mathrm{c})$ porte trois négatifs de supports qui n'ont pas été retrouvés. A2\#22 et $\mathrm{B} 1 \# 93$ ont la particularité d'être dans une matière première à grain très fin, « cireux ».

Les remontages B1\#107 (fig. 7a) et surtout A2\#56 (fig. 6) concernent une production laminaire. Cette phase est repérable par le soin apporté à la préparation du point d'impact : dégagement par enlèvement de petits éclats allongés ou rebroussés sans doucissage ou abrasion. II a d'ailleurs été possible de remonter l'esquille de préparation du talon B1\#289 sur le remontage A2\#56. Les talons sont 


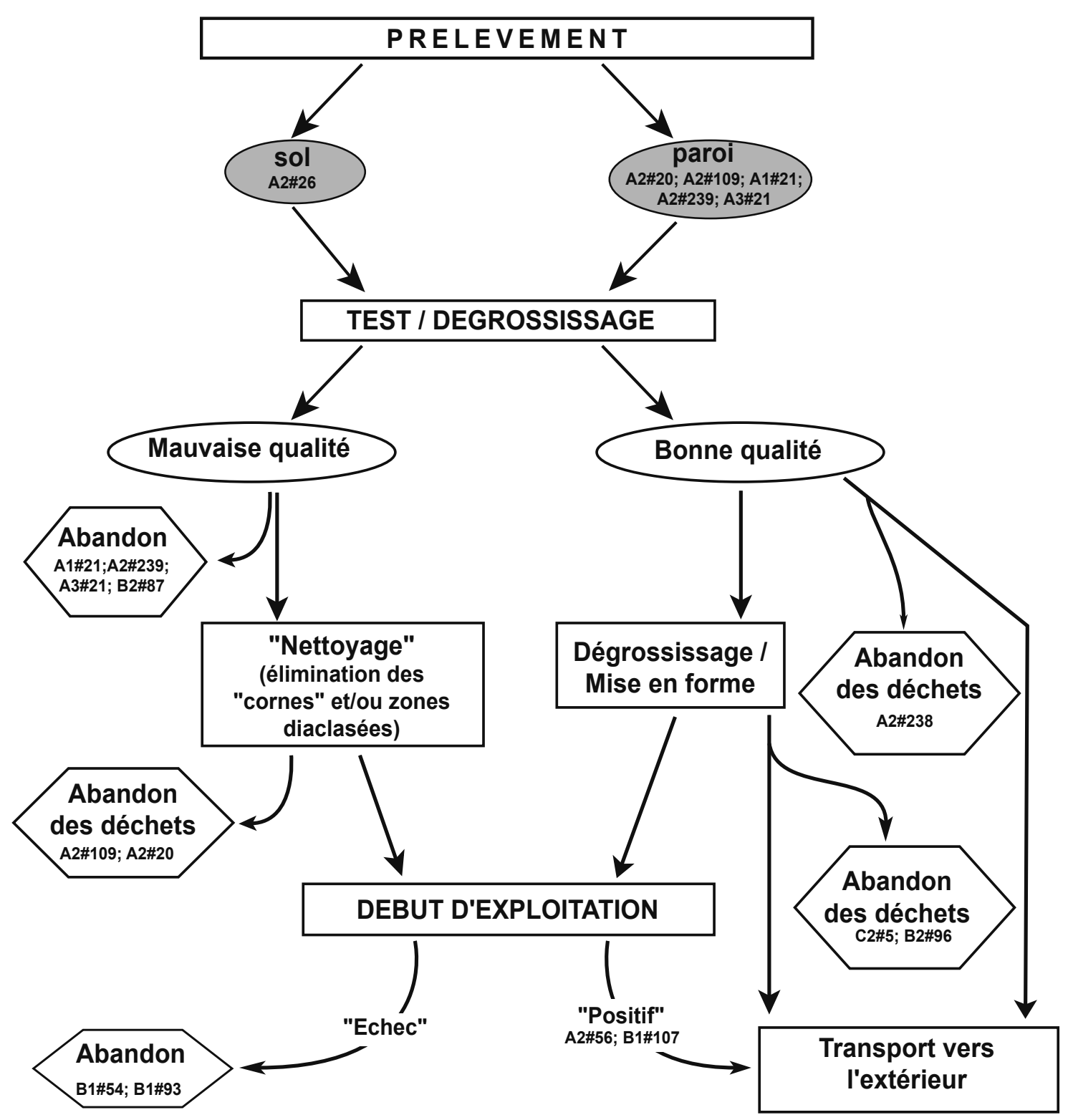

Figure 4 - Chaîne opératoire de l'exploitation du silex.

Figure 4 - "Chaîne opératoire" of flint reduction.

donc généralement de dimension plus réduite, voire punctiformes. La mise en forme des blocs peut être minimale puisque l'une des lames du remontage B1\#59 (fig. 5b) est semi-corticale. À l'inverse, le remontage A2\#56 (fig. 6) indique l'exploitation d'une surface très plane, bordée par une crête latérale.

Le remontage de B1\#107 (fig. 7a) est accompagné de nombreux restes appartenant au même bloc mais sans que des raccords physiques aient pu être réalisés (B1\#217, B1\#179, B1\#106 mais aussi B2\#22 qui sont des esquilles bulbaires ou des éclats de flancs).

\subsection{6 - Conclusions}

Si les remontages démontrent que certains des nodules taillés ont pu être prélevés sur la paroi, d'autres blocs ont dû être récoltés sur le sol ou extraits du remplissage argileux. Ce dernier cas est sans doute illustré par l'élargissement important dans le carré A2 de la fente de retrait qui recoupe la zone fouillée. Insistons aussi sur le fait que les remontages avec les négatifs en paroi ont été positifs uniquement dans le secteur immédiatement susjacent à la zone fouillée alors qu'ils ont été tentés sur les deux parois de la galerie et sur une trentaine de mètres. 


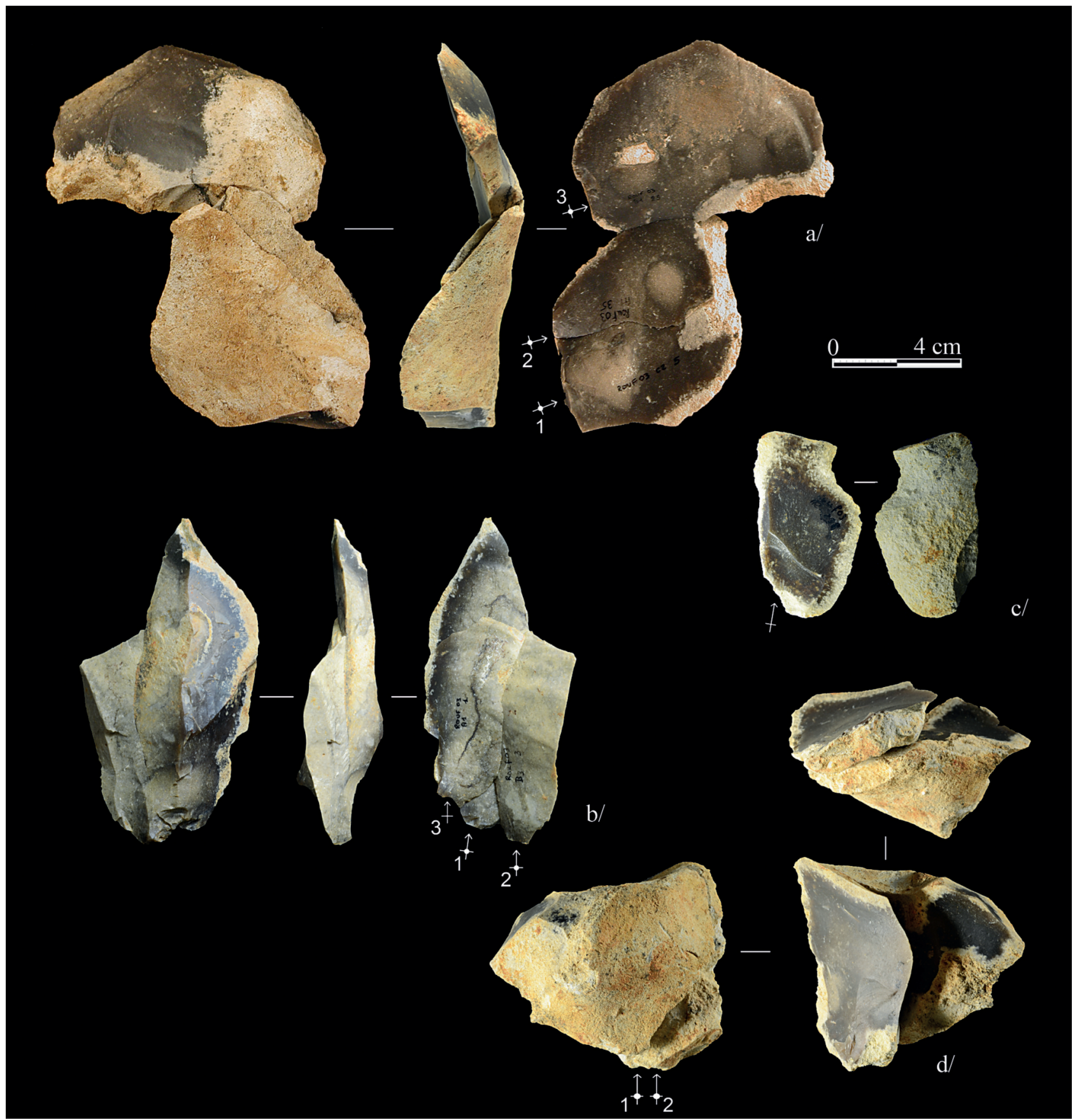

Figure 5 - a- C2\#5. Série de trois éclats de dégrossissage ; matériau à grain fin, cireux. b- B1\#59. Série de trois éclats de dégrossissage/cintrage d'un nucléus à lame (noter la préparation du talon). Le troisième éclat a un talon écrasé. c - A2\#238. Éclat cortical à grain cireux. Talon absent. $d$ - B2\#96. deux éclats de dégrossissage ; matériau à grain fin, cireux.

Figure 5 - a- C2\#5. Series of three roughing-out flakes; Fine-grained waxy flint. b-B1\#59. Series of three roughing-out/cintrage flakes on a blade core (note the preparation of the butt). The third flake has crushed butt. c-A2\#238. Cortical flake. $d$ - B2\#96. two roughing-out flakes. Fine-grained waxy flint. 


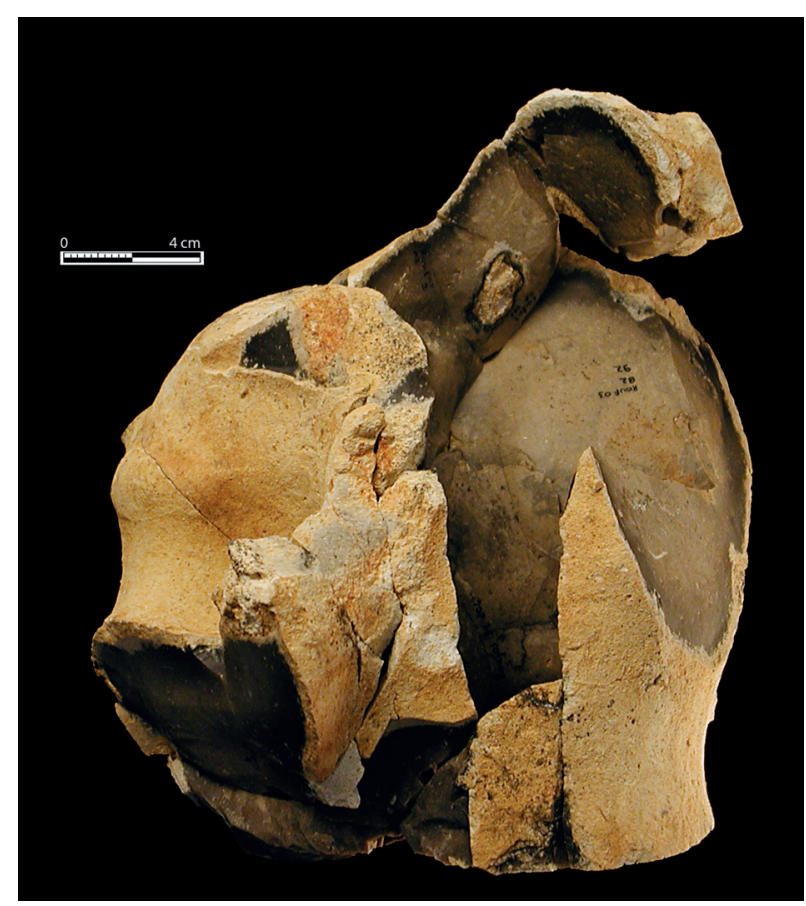

Figure 6 - A2\#56. Dégrossissage d'un nucléus à lame et début d'exploitation (non visible sur la photo).

Figure 6 - A2\#56. Roughing-out of a blade core and beginning of reduction (not visible in the photo).

Cet ensemble très homogène dans sa gestion et dans ses modes de percussion montre que les activités pratiquées sur place sont exclusivement de la taille. L'absence totale d'outillage, déjà perçue à la fouille, est confirmée. Non seulement l'outillage « classique » détectable à ses retouches plus ou moins abruptes n'existe pas dans la série, mais il n'y a pas davantage de pièces utilisées, ou mâchurées. Les seules traces de grignotages ou de " retouches " sont rapportables à des accidents de taille (voir par exemple A2\#37). Notons aussi l'absence de percuteur, en roche allochtone comme sous forme de nodule de silex. En effet, aucun de ces derniers ne porte de stigmates attribuables à cette utilisation.

L'étude systématique de ce matériel démontre la présence sur place des trois premières étapes de la chaîne opératoire d'exploitation de la matière première : le test de blocs, le dégrossissage et l'ébauche d'une production sur place (lamelles notamment) reconnaissable au soin apporté dans la préparation des talons. Elles renvoient aussi à plusieurs comportements liés à la fois à la qualité de la matière première et aux objectifs du/des tailleur(s). Ainsi rencontre-t-on des blocs testés et abandonnés parce que de mauvaise qualité ; des blocs testés et emportés (ne subsistent alors que des éclats épars qui reflètent souvent la bonne qualité du matériau) ; des blocs testés, dégrossis puis emportés (nous disposons alors de séries d'éclats corticaux formant l'enveloppe du nodule) ; enfin, il y a les blocs testés, dégrossis, puis exploités sur place. Bien que cette situation ne soit que peu documentée dans le matériel, elle reflète un comportement difficile à comprendre dans un environnement inconfortable, voire hostile, par comparaison avec le porche d'entrée baigné de lumière du jour.

\section{4 - Répartition spatiale des vestiges}

Rappelons que le locus étudié a des dimensions réduites (env. $3 \mathrm{~m}^{2}$ ) au regard des galeries de Rouffignac. L'étude de la répartition spatiale des vestiges prélevés n'a, dans ce contexte, qu'une valeur d'échantillon.

Cependant, la lecture de la figure 9 amène quelques commentaires. Le premier est relatif à la densité des vestiges d'un sous-carré à l'autre. Les grands éléments sont regroupés dans le fond de la cuvette de B2S3 (par gravité) et le long de la paroi (y compris sous le redan qu'elle forme près du sol). Toutefois, leur position est peu significative, parce qu'ils ont pu subir des déplacements au fil du temps, à cause de la fréquentation ancienne et répétée des galeries. Au contraire, les petits éléments sont absents de B2S3 (cuvette naturelle, dont le fond est induré par rubéfaction) où a sans doute été installé un éclairage. Ces mêmes petits éléments sont très nombreux en B1S4, et près de la paroi en A2S4. Enfin, la densité de vestiges est faible en B1S2 et B2S2/B3S1.

D'un autre point de vue, la figure 9a présente la répartition de vestiges participant au remontage du bloc B2\#87 (test très poussé d'un bloc de mauvaise qualité dont tous les produits sont abandonnés sur place). II montre que les vestiges concernés n'ont pas subi de déplacements importants dans l'axe de la galerie, mais plutôt transversalement. Le constat est sensiblement le même pour le remontage A2\#56 (fig. 9b).

\section{5 - Datations radiocarbone}

Une cinquantaine de charbons ont été prélevés. Ils sont de deux types bien différents. Les premiers se présentent comme des brindilles ou des pailles et nous sont apparus par-dessus les vestiges lithiques. Les seconds sont massifs et étaient plutôt concentrés dans la cuvette peu profonde du carré B2, secteur 3. L'analyse anthracologique des échantillons datés, réalisée par St. Thiébault, montre que les brindilles appartiennent à une Rosacées alors que les charbons plus massifs correspondent au Noisetier. La présence de ces deux types d'échantillons a conduit à la réalisation de deux datations radiocarbone par AMS au Laboratoire des Sciences du Climat et de l'Environnement. Les analyses faites sur Artémis (LMC14-Saclay) documentent deux fréquentations distinctes de la caverne. La Rosacée (A1 n 30, GifA 70008/SacA6412) est datée de $2800 \pm 35$ ans BP qui, une fois calibré, donne l'intervalle 1 043-846 ans cal BC (à 95,4\% de chance). Quant au charbon de noisetier (B2 $n^{\circ}$ 312, GifA 70007/SacA6411) de quelques millénaires plus anciens, il est daté de $8530 \pm$ 50 ans BP ce qui correspond à l'intervalle calibré 7 6087505 cal BC (Bronk Ramsey 2013 ; Reimer et al. 2013). 


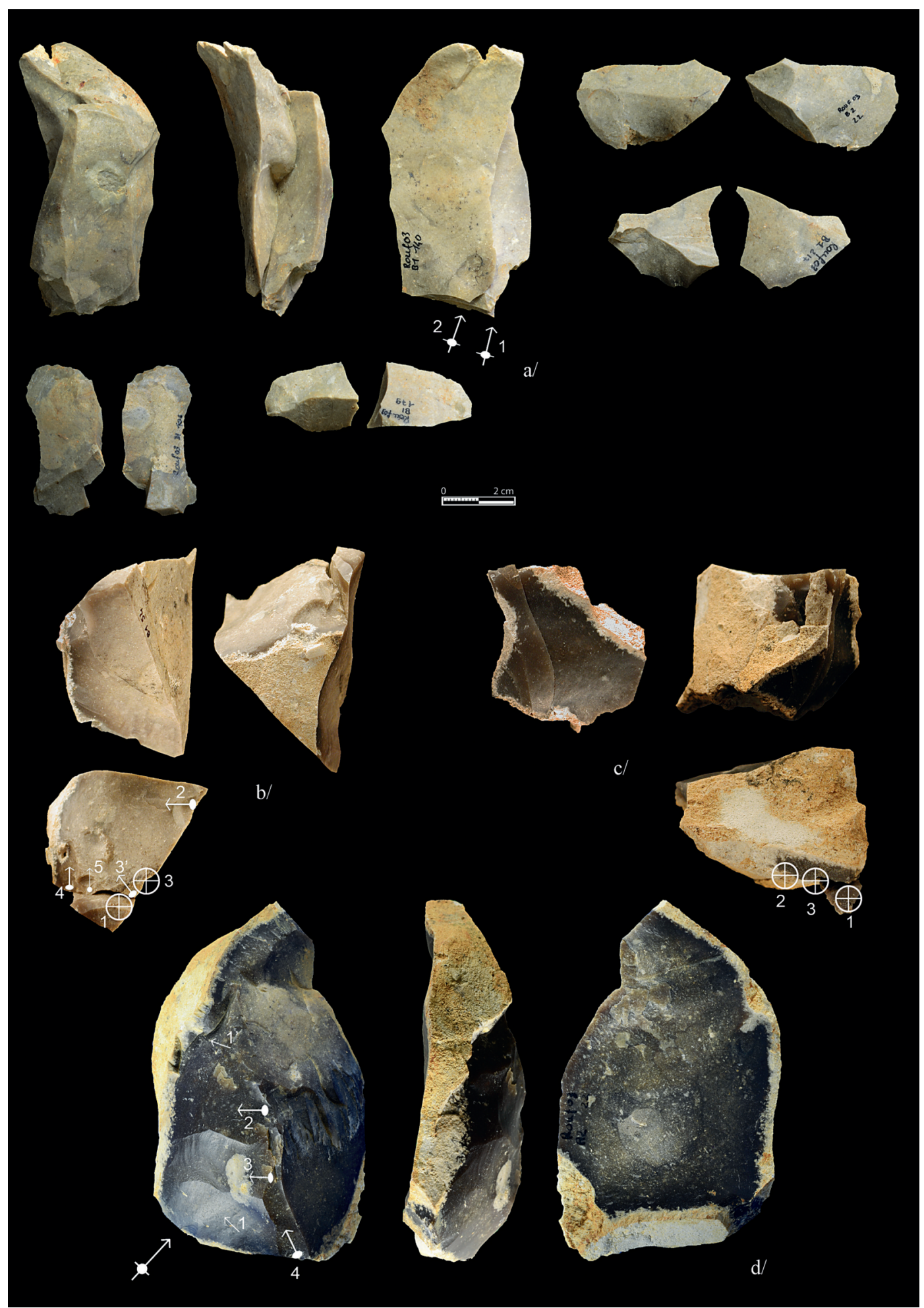

Figure 7 - Exemples de production laminaire et lamellaire. a- Ensemble d'éclats et remontage B1\#107 : ces éléments correspondent à la préparation et au début de l'exploitation d'un nucléus à lames. b- A1\#38: Nucléus à lamelles avec production d'une lamelle semi-corticale (1) puis préparation d'un nouveau plan de frappe (2, 3', 4 et 5). c- B1\#93: Nucléus à lamelles à grain cireux. Entame avec un éclat laminaire (1) puis obtention d'un second éclat laminaire dont seule la partie distale a été retrouvée (2) puis production d'une lamelle non retrouvée (3). Le plan de frappe est diaclasique. $d$ - A2\#22 : Ebauche de nucléus à lamelles en silex à grain cireux : préparation d'une crête centrale à 1 pan $(1,1,2$ et 3) puis production d'une lamelle non retrouvée (4).

Figure 7 - Example of blade and bladelet production. a- Flakes and refit B1\#107: preforming and beginning of blade production. $b$ - A1\#38: bladelet core; production of semi-cortical bladelet (1), followed by the preparation of a new striking platform (2, 3', 4 and 5). c- B1\#93: bladelet core, waxy flint. Description of the refitting: Elongated flake (1) is removed, then a second elongated flake (2) is removed (only the distal portion was recovered) and a bladelet, is removed, also not recovered. The striking platform is a natural surface. $d$ - A2\#22: roughing out of a bladelet core, waxy flint. Preparation of a central crest (1, 1,2 and 3) then production of a bladelet that was not recovered (4). 


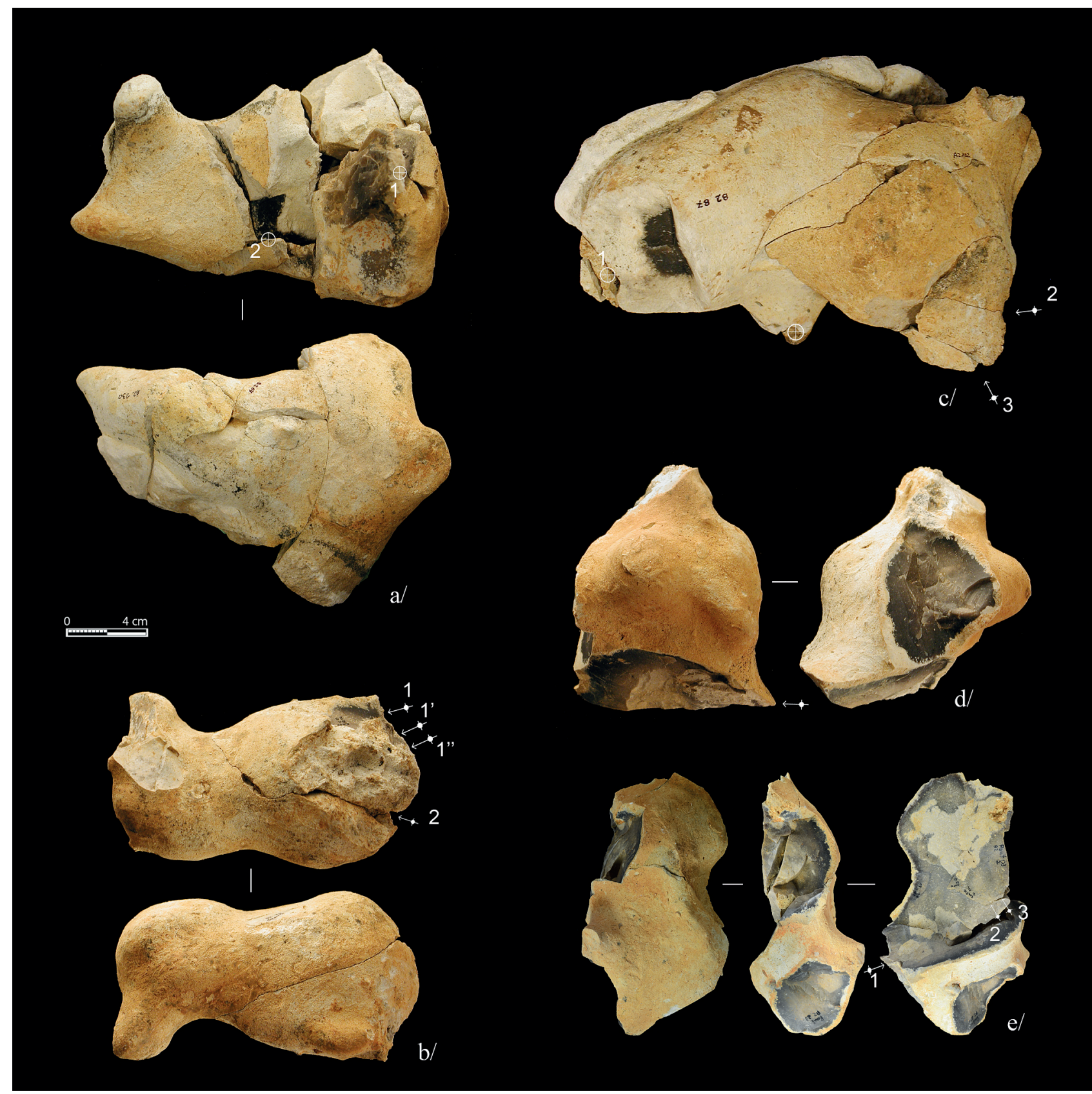

Figure 8 - Exemples de tests de blocs. a- A2\#233 : 2 zones d'impact. b- A2\#26 : 2 zones d'impact. c- B2\#87 : 3 zones d'impact. Les directions 2 et 3, perpendiculaires, sont utilisées en alternance. $d$ - A2\#239 : 1 point d'impact. e- A2\#20 : Remontage de 3 éclats (dont 1 avec cassure Siret).

Figure 8 - Examples of tested blocks. a- A2\#233: 2 impact areas. b- A2\#26: 2 impact areas. c- B2\#87: 3 impact areas. The directions 2 and 3 (perpendicular) are used alternately. $d$ - A2\#239: 1 impact area. e- A2\#20: refitting of 3 flakes (1 with Siret fracture).

\section{6 - Interprétations}

La première des deux datations obtenues (1 043-846 ans cal $\mathrm{BC}$ ) renvoie à la fin de l'Âge du Bronze. Ce résultat est cohérent avec la présence de dépôts de cendres associés à des vestiges céramiques classiquement attribués au Bronze final (Barrière 1974a et b ; Chevillot 1981), à proximité du locus fouillé. Cependant, l'absence d'indice de percussion au métal, la position de la brindille datée (audessus des vestiges lithiques) et la finalité sépulcrale des fréquentations protohistoriques de la cavité n'incitent pas à rapporter l'exploitation du silex aux Ages des Métaux.

Le second échantillon daté fournit, pour sa part, un résultat (7 608-7 505 ans cal BC) qu'il est facile de rapprocher des occupations mésolithiques du porche. Entre 1957 et 1962, 

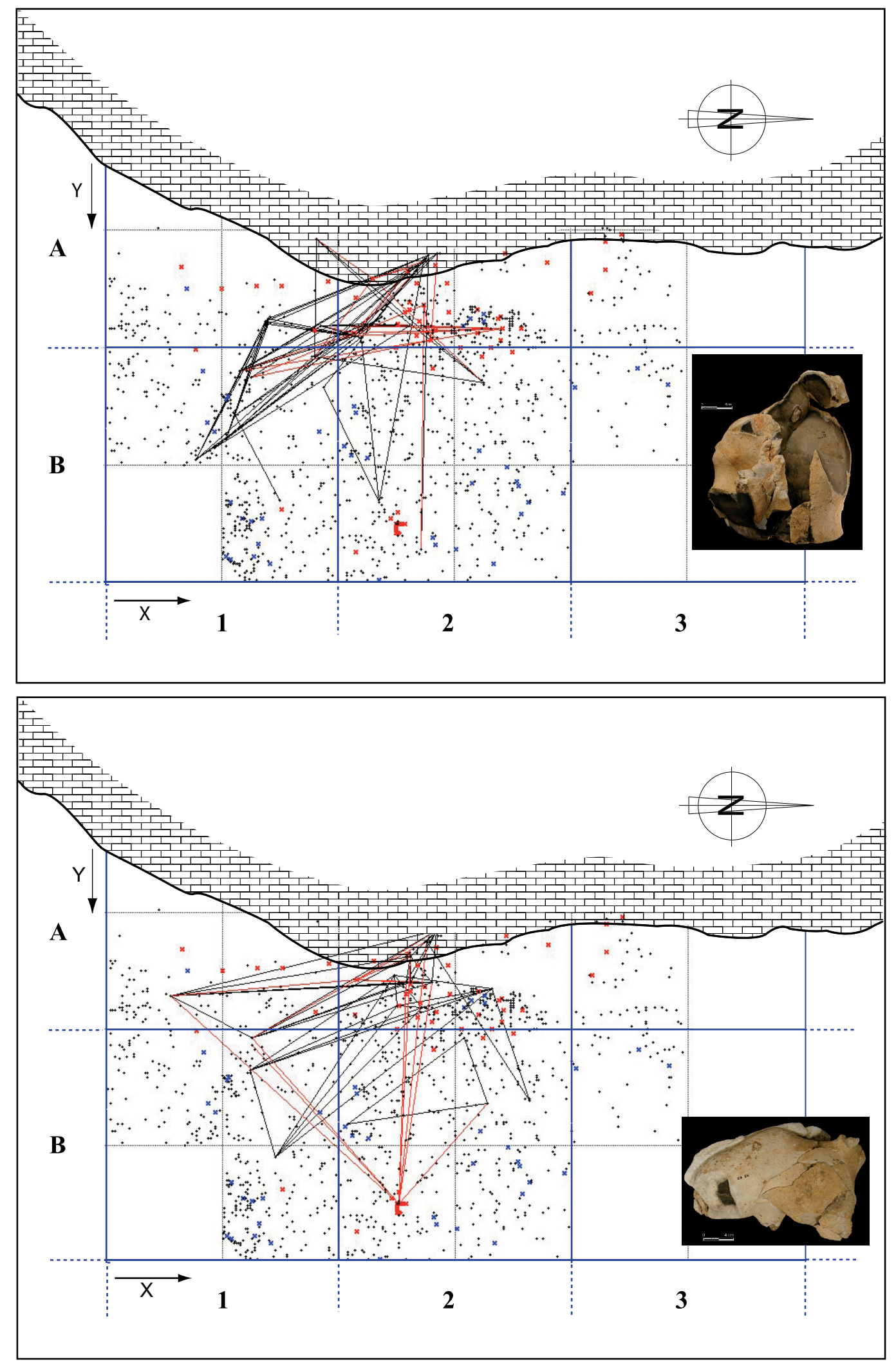

Figure 9 - Répartition spatiale des vestiges cotés. En rouge, les pièces de dimension supérieure à $5 \mathrm{~cm}$, en bleu, les pièces de dimensions inférieures à $1 \mathrm{~cm}$, en noir, les autres. a-remontage A2\#86. b-remontage B2\#87. (Projection L. Daulny).

Figure 9 - Spatial distribution of piece-plotted artefacts. In red, artefacts greater than $5 \mathrm{~cm}$. In blue, artefacts smaller than $1 \mathrm{~cm}$. In black, remaining pieces. a- Refit A2\#86. b- Refit B2\#87. (Projection L. Daulny). 
celui-ci $\mathrm{a}$, en effet livré à $\mathrm{Cl}$. Barrière une séquence importante (couches 5 à 2 , et leurs subdivisions) documentant des occupations qualifiées alors de sauveterriennes et tardenoisiennes (tabl. 1), et rapportables au premier et au second Mésolithique (Barrière 1973a et b). La couche 4 donne des datations radiocarbone équivalentes à celle du locus étudié ici (fig. 10). L'hypothèse d'une exploitation de la matière première siliceuse de la grotte au cours du premier Mésolithique s'en trouve confortée, d'autant que les cortex observables sur de nombreuses pièces de la couche 4 présentent les mêmes caractéristiques que les nodules du secteur fouillé.

L'utilisation de la percussion dure lors des phases de test et de dégrossissage des matériaux est un choix technique ubiquiste qui ne peut être utilisé comme argument chronologique. En revanche, le relatif soin apporté à la préparation du talon, la robustesse des supports laminaires et l'utilisation très probable de la percussion minérale dure au cours des phases de production s'accordent mieux avec le premier Mésolithique qu'avec le Magdalénien récent ou le second Mésolithique.

Rappelons que la surface étudiée ici est réduite au regard de l'étendue de la caverne. Elle l'est aussi par rapport à l'extension probable des zones ayant conservé des traces d'exploitation du silex. Celles-ci semblent très nombreuses au-delà du locus étudié, dans les galeries $\mathrm{H}$ et I, comme audelà du Grand plafond. Dans ce dernier cas, une galerie basse (environ $1 \mathrm{~m}$ de haut), conserve plusieurs petits amas ( $1 \mathrm{~m}^{2}$ chacun) et présentent localement des nappes de vestiges manifestement moins bien conservés (fig. 11). Au total, ce sont plusieurs centaines de mètres carrés de sols qui, à des degrés divers de préservation, pourraient documenter l'exploitation des silex de la grotte.

À l'échelle du site, notre étude établit que l'exploitation du silex de la grotte, quoique envisagée par $\mathrm{Cl}$. Barrière, a jusqu'à présent été largement sous-estimée. Mais ce constat amène à une autre remarque. Pour de nombreux auteurs, l'une des caractéristiques des assemblages lithiques mésolithiques de Rouffignac est le faible taux de microlithes. Les méthodes de prélèvement ne peuvent à elles seules justifier ce fait. En revanche, la présence d'une matière première abondante et disponible explique sans doute l'abondance des produits de débitage. II serait néanmoins abusif de réinterpréter les occupations du porche en insinuant que c'est cette matière première qui, à elle seule, justifie l'installation des Hommes à cet endroit. La richesse du mobilier archéologique, comprenant toutes les catégories de vestiges y compris industrie osseuse et art mobilier (Marquebielle 2014), montre bien que le porche de Rouffignac n'a pas été un site spécialisé, dédié uniquement à l'exploitation du silex.

Un autre enseignement à l'échelle du site réside dans la nécessité d'une réévaluation du potentiel archéologique des sols des galeries de Rouffignac, trop longtemps considérés comme trop perturbés par les fréquentions historiques pour avoir un réel intérêt. Bien que la lecture des indices archéologiques soit délicate, et que leur

\begin{tabular}{|c|c|c|c|c|c|}
\hline Stratigraphie & Attribution & Ref. Labo & Matériaux/ méthode & Âge BP & $\begin{array}{c}\text { Âge cal BC } \\
\text { à } 95,4 \% \text { de chance }\end{array}$ \\
\hline Couche 2 & Second & GrN-5512 & Charbon / comptage & $6400 \pm 40$ & $5471-5317$ \\
\hline Couche 3 & Mésolithique & Gro-2889 & Charbon / comptage & $7800 \pm 50$ & $6767-6496$ \\
\hline Couche 4 a & \multirow{6}{*}{$\begin{array}{c}\text { Premier } \\
\text { Mésolithique }\end{array}$} & Gro-2913 & Charbon / comptage & $8370 \pm 100$ & $7589-7145$ \\
\hline Couche $4 \mathrm{~b}$ & & Gro-2895 & Charbon / comptage & $8590 \pm 95$ & $7940-7484$ \\
\hline Couche $4 \mathrm{c}$ & & Gro-2880 & Charbon / comptage & $8995 \pm 105$ & $8451-7795$ \\
\hline Couche 5 a & & GrN-5513 & Charbon / comptage & $8750 \pm 75$ & $8187-7596$ \\
\hline Couche $5 \mathrm{~b}$ & & GrN-5514 & Charbon / comptage & $9150 \pm 90$ & $8611-8236$ \\
\hline Atelier (B2\#312) & & GifA 70007 & Charbon / AMS & $8530 \pm 50$ & $7608-7505$ \\
\hline
\end{tabular}

Tableau 1 - Datations radiocarbone des occupations mésolithiques de Rouffignac (d'après Barrière 1973b pour les niveaux du porche d'entrée). Les âges C-14 sont calibrés avec la courbe IntCal 2013 (Reimer et al. 2013) et le logicial Oxcal v4.2.4 (Bronk Ramsey 2013). Les âges calibrés sont donnés à deux sigma (95,4\% de chance).

Tableau 1 - Radiocarbon dating of the Mesolithic occupations of Rouffignac (after Barrière 1973b for the levels at the entrance). The C-14 dates are calibrated using IntCal 2013. (Reimer et al. 2013) and the software OxCal v.4.2 (Bronk Ramsey 2013). The Calibrated intervals are given with a confidence level of $95.4 \%$. 


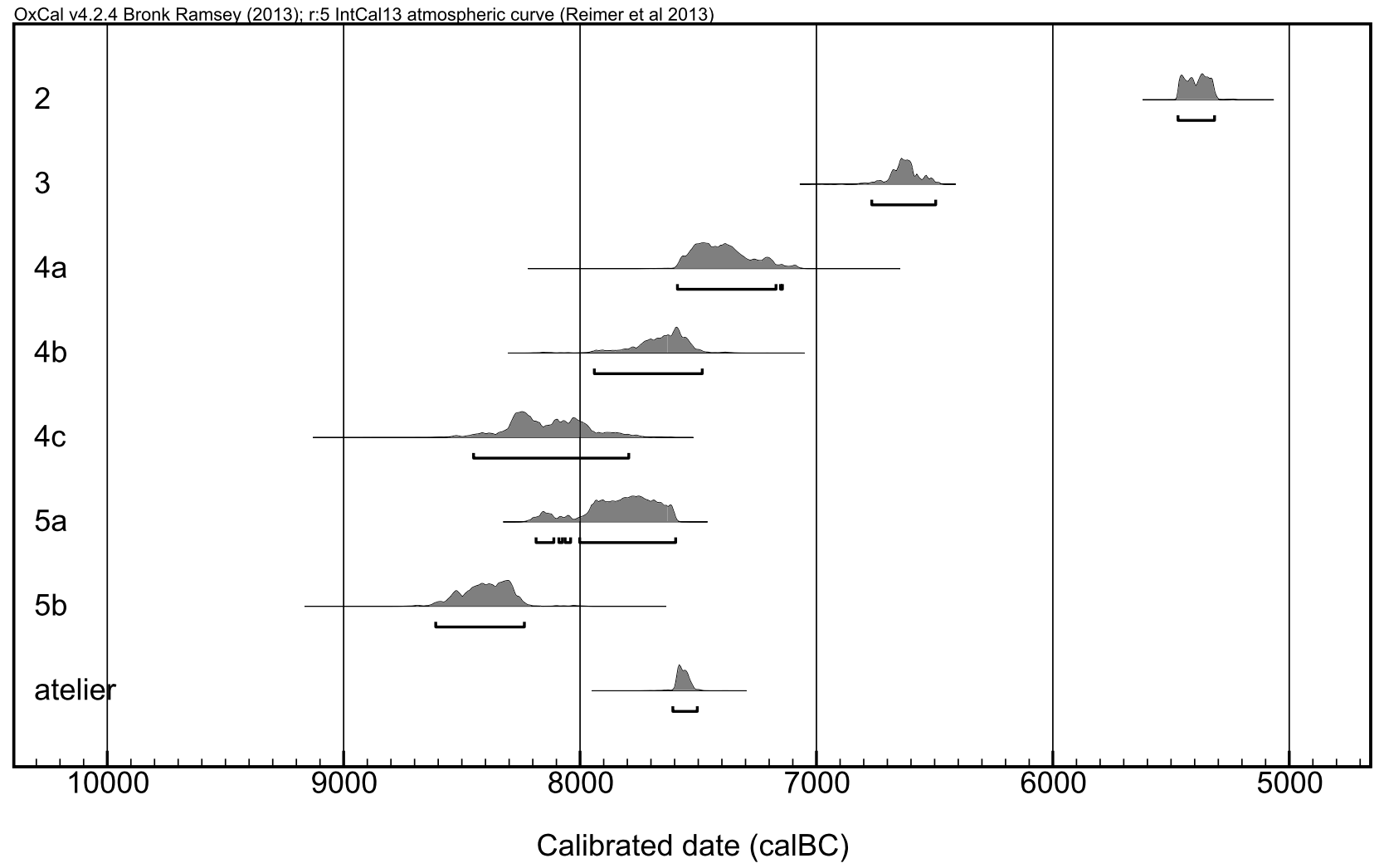

Figure 10 - Les datations radiocarbone des échantillons mésolithiques (tabl.1) de Rouffignac sont calibrées avec IntCal 2013 (Reimer et al. 2013) et le logiciel OxCal v.4.2 (Bronk Ramsey 2013). Les âges calibrés sont donnés à deux sigma (95,4\% de chance). La date obtenue sur le charbon du locus étudié concorde avec celles des couches 4.

Figure 10 - Radiocarbon dating of the Mesolithic samples of Rouffignac (tabl. 1) are calibrated using IntCal 2013 .(Reimer et al. 2013) and the software OxCal v.4.2 (Bronk Ramsey 2013). Calibrated results are given at 2 sigma (95.4\% of confidence level). The date obtained on the charcoal from the study area is consistent with those from layers 4.

attribution chronologique soit souvent sujette à discussion, il est clairement exagéré d'abandonner tout espoir de découvrir localement des documents exploitables, relatifs à telle ou telle fréquentation préhistorique de la caverne.

Séquence chrono-culturelle incontournable du Mésolithique aquitain (Rozoy 1978 ; Valdeyron 1994 ; Roussot-Larroque 2009), Rouffignac livre à présent aussi le témoignage d'un comportement économique inédit. L'exploitation en priorité de matières premières locales est privilégiée à cette période (Eriksen et Fisher 2002), mais elle prend ici une dimension particulière puisqu'elle s'étend à un milieu naturel a priori hostile : des galeries naturelles profondes. Celles-ci cependant ne semblent pas avoir accueilli d'autres activités que celles décrites ici. Les contraintes du milieu ont manifestement induit une spécialisation de l'aire d'activité bien plus stricte que sur des sites d'atelier de plein-air.

\section{Remerciements}

Treize années se sont écoulées depuis l'opération de terrain à l'origine de cet article. Au fil du temps, nous avons reçu l'aide de quelques amis et collègues : P.-Y. Demars fut le premier à regarder le matériel lithique; C. Ferrier consacra un peu de temps à valider, sur la base d'un prélèvement micromorphologique, la présence du foyer d'éclairage ; L. Daulny réalisa l'analyse de la répartition spatiale des vestiges; $\mathrm{S}$. Thiébault fit la détermination anthracologique des charbons destinés à la datation radiocarbone ; B. Gravina a bien voulu assurer la relecture (et un peu plus !) du résumé et des légendes en anglais. En outre, ayant présenté cette étude à la table-ronde "Des techniques aux territoires : nouveaux regards sur les cultures mésolithiques " à Toulouse en 2012, nous avons pu profiter de fructueux échanges avec les spécialistes du Mésolithique ; échanges qui se sont prolongés avec les relecteurs de cet article. Que tous trouvent ici l'expression de nos sincères remerciements.

Le Service Régional d'Archéologie d'Aquitaine a financé cette opération et les datations radiocarbone qui y ont fait suite. Merci à D. Barraud et J.-M. Geneste alors en charge de ce dossier pour leur soutien et leur confiance. 

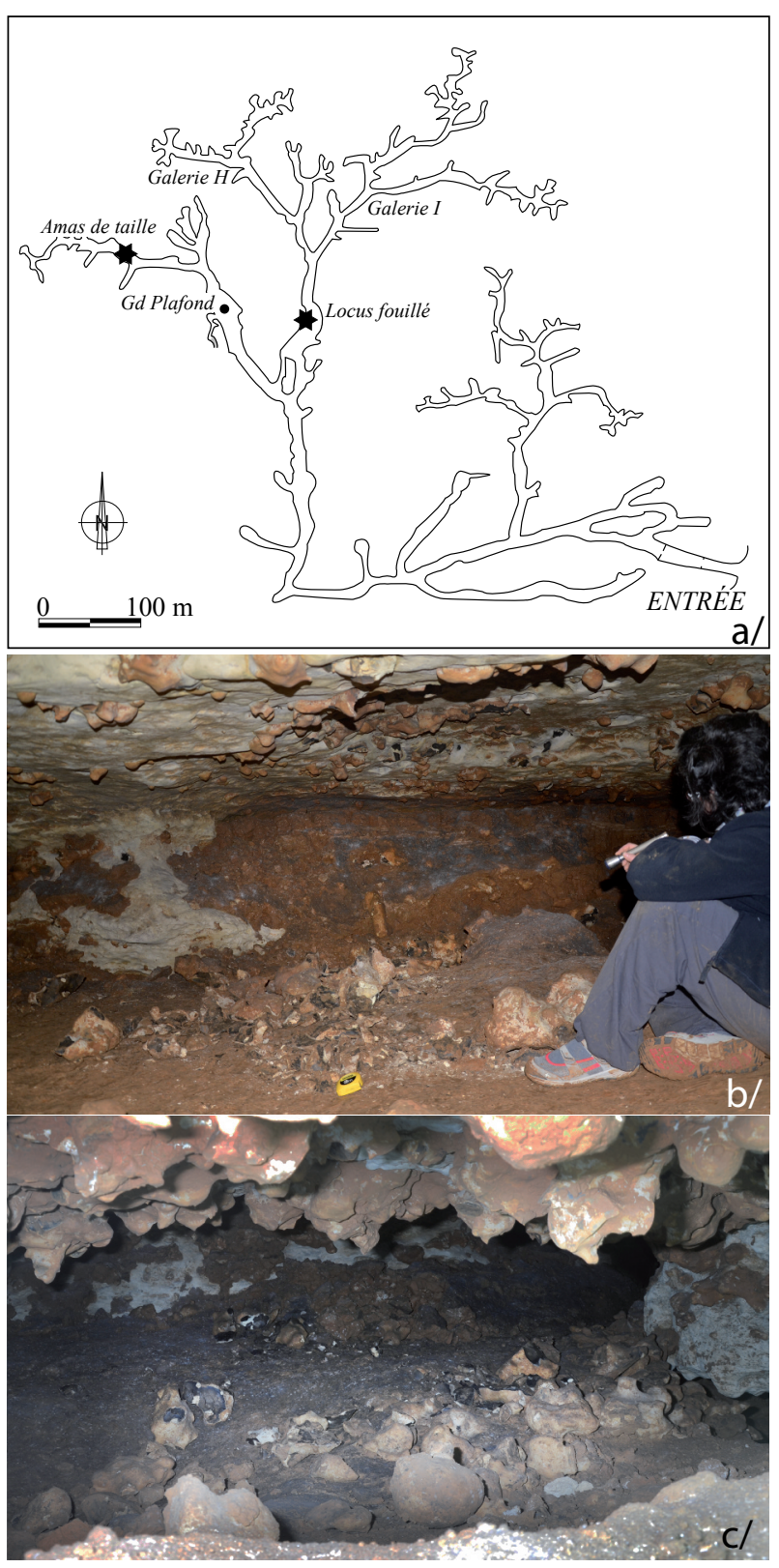

Figure 11 - a- Localisation des amas de taille évoqués dans l'étude. Carte non exhaustive. b et c- amas de taille au-delà du Grand Plafond, dans une galerie dont la hauteur ne dépasse pas $1 \mathrm{~m}$.

Figure 11 - a- Location of the knapping clusters mentioned in the text (not exhaustive map). $b$ - and c-knapping cluster in a low gallery beyond the Great Ceiling (maximum height : $1 \mathrm{~m}$ ).

\section{Références bibliographiques}

APELLANIZ J.-M., DOMINGO MENA 1987 - Estudio sobre Atapuerca (Burgos). II-Los materiales de superficie del sanctuario de la galeria del silex. Estudios de arqueologia de Deusto, $342 \mathrm{p}$.

BARRIÈRE Cl. 1973a - Rouffignac, l'archéologie. Mémoire de l'Institut d'art préhistorique II, fascicule 1. Publication de l'Institut d'art préhistorique de I'Université Toulouse-LeMirail, $160 \mathrm{p}$.

BARRIÈRE Cl. 1973b - Rouffignac, l'archéologie. Mémoire de l'Institut d'art préhistorique II, fascicule 2. Publication de l'Institut d'art préhistorique de I'Université Toulouse-LeMirail, $210 p$.

BARRIÈRE Cl. 1974 - Rouffignac, l'archéologie. Mémoire de l'Institut d'art préhistorique II, fascicule 3. Publication de l'Institut d'art préhistorique de I'Université Toulouse-LeMirail, $83 \mathrm{p}$.

BARRIÈRE Cl. 1982 - L'art pariétal de Rouffignac, Picard, $208 \mathrm{p}$.

BRONK RAMSEY C., SCOTT M., van der PLICHT J. 2013 - Calibration for archaeological and Environmental Terrestrial Samples in the Time Range 26-50 ka cal BP. Radiocarbon 55(4), p. 2021-2027.

CHEVILLOT Ch. 1981 - La civilisation de la fin de l'Age du Bronze en Périgord, Archéologie, recherche et civilisation, $221 \mathrm{p}$.

ERIKSEN B. V., FISHER L. E. 2002 - Lithic raw material economy in Late Glacial and Early Postglacial western Europe : Introduction. In: Fisher et Eriksen B. V. (eds) : Lithic raw material economies in Late Glacial and Early Postglacial Europe. BAR International series 1093, p. 1-5.

GOPHER A., BARKAI R. 2006 - Flint Extraction Sites and Workshops in Prehistoric Galilee, Israel. In : Gabriele Körlin, Gerd Weisgerber (dir) Stone Age - Mining Age - Der Anschnitt, Beiheft 19, p. 91-98.

MARQUEBIELLE B. 2014 - Le travail des matières osseuses au Mésolithique : caractérisation technique et économique à partir des séries du Sud et de l'Est de la France. Thèse de Doctorat de l'Université de Toulouse-IILe-Mirail, 508 p.

NOUGIER L.-R., ROBERT R. 1957 - Rouffignac ou la Guerre des mammouths, Paris, Editions de La Table Ronde, 311p.

PLASSARD F. 2005a - Les grottes ornées de Combarelles, Font-de-Gaume, Bernifal et Rouffignac. Contexte archéologique, thèmes et style des représentations. Thèse de l'Université Bordeaux 1, $413 \mathrm{p}$.

PLASSARD F. 2005b - Grotte de Rouffignac. Fouille au pied d'une paroi ornée. Bilan scientifique 2003 du Service Régional de l'Archéologie Aquitaine, p. 36. 
PLASSARD J. 1999 - Rouffignac, le sanctuaire des mammouths. Paris, Le seuil. 96 p., 96 fig.

PLATEL J.-P. 1999 - Thenon. Carte Géologique de la France à 1/50 000. Éditions du BRGM.

REIMER P. J., BARD E., BAYLISS A., BECK J. W., BLACKWELL P. G., BRONK RAMSEY C., BUCK C. E., CHENG H., EDWARDS L., FRIEDRICH M., GROOTES P. M., GUILDERSON T.P., HAFLIDASON H., HAJDAS I., HATTÉ C., HEATON T., HOFFMANN D. L., HOGG A. G., HUGHEN K. A., KAISER K. F., KROMER B., MANNING S. W., NIU M., REIMER R. W., RICHARDS D. A. SCOTT E. M., SOUTHON J. R., STAFF R. A., TURNEY C. S. M., van der PLICHT J. 2013 - IntCal13 and Marine 13 Radiocarbon age calibration curves 0-50.000 years cal BP. Radiocarbon 55(4), p. 1869-1887.
ROUSSOT-LARROQUE J. 2009 - Les complexes géométriques en Aquitaine In : El Mesolítico Geométrico en la Península Ibérica. Monografías Arqueológicas 44, p. 279-306.

ROZOY J. G. 1978 - Les derniers chasseurs. L'Epipaléolithique en France et en Belgique. Charleville, $\mathrm{n}^{\circ}$ spécial du Bulletin de la Société Archéologique Champenoise, $1257 \mathrm{p}$.

VALDEYRON N. 1994 - Le Sauveterrien : cultures et Sociétés mésolithiques dans la France du sud durant Le $X^{e}$ et le $I X^{e}$ millénaire $B P$, Thèse de doctorat, Toulouse, Université Toulouse-II-Le-Mirail, 2 vol., 584 p. 